\title{
Влияние температуры спекания на термоэлектрические свойства соединения $\mathrm{Bi}_{1.9} \mathrm{Gd}_{0.1} \mathrm{Te}_{3}$
}

\author{
(C) М.Н. Япрынцев ${ }^{1}$, А.Е. Васильев ${ }^{1}$, О.Н. Иванов ${ }^{1,2}$ \\ ${ }^{1}$ Белгородский государственный национальный исследовательский университет, \\ 308015 Белгород, Россия \\ ${ }^{2}$ Белгородский государственный технологический университет им. В.Г. Шухова, \\ 308012 Белгород, Россия \\ E-mail: yaprintsev@bsu.edu.ru
}

(Поступила в Редакцию 20 декабря 2018 г.

В окончательной редакции 24 декабря 2018 г.

Принята к публикации 28 декабря 2018 г.)

\begin{abstract}
Изучены закономерности влияния температуры спекания (750, 780, 810 и $840 \mathrm{~K})$ на элементный состав, параметры кристаллической решетки, удельное электрическое сопротивление, коэффициент Зеебека, полную теплопроводность и термоэлектрическую добротность соединения $\mathrm{Bi}_{1.9} \mathrm{Gd}_{0.1} \mathrm{Te}_{3}$. Установлено, что в процессе высокотемпературного спекания элементный состав образцов изменяется вследствие интенсивного испарения теллура, что может приводить к формированию различных точечных дефектов (вакансий и антструктурных дефектов), влияющих на концентрацию и подвижность основных носителей заряда (электронов). Температура спекания сильно влияет на удельное электрическое сопротивление образцов, тогда как влияние на коэффициент Зеебека и полную теплопроводность выражено гораздо слабее. Наибольшее значение термоэлектрической добротности $(Z T \approx 0.55)$ наблюдалось для образца, спеченного при температуре $750 \mathrm{~K}$.
\end{abstract}

DOI: $10.21883 /$ FTP.2019.05.47550.08

\section{1. Введение}

Известно, что эффективность термоэлектрических материалов определяется коэффициентам термоэлектрической добротности $(Z T)$, который может быть выражен как $\left(S^{2} / \rho \kappa\right) T$, где $T-$ абсолютная температура, $\mathrm{S}-$ коэффициент Зеебека, $\rho$ - удельное электрическое сопротивление и $\kappa-$ полная теплопроводность [1].

В настоящее время теллурид висмута $\mathrm{Bi}_{2} \mathrm{Te}_{3}$ и соединения на его основе являются наиболее распространенными низкотемпературными термоэлектрическими материалами, поскольку они имеют самые высокие значения ZT при температурах вблизи комнатной [2]. Однако термоэлектрическая добротность этих материалов все еще слишком мала $(Z T<1)$, что делает их использование нерентабельным в большинстве приложений. Для улучшения термоэлектрических характеристик соединений на основе $\mathrm{Bi}_{2} \mathrm{Te}_{3}$ используются различные физические и технологические подходы [3-10]. Из этих подходов легирование является одним из перспективных и эффективных способов оптимизации термоэлектрических параметров $(S, \rho, \kappa)$ [11-16].

Как было недавно установлено [17-25], в качестве эффективных легирующих примесей могут быть использованы редкоземельные элементы $(R=\mathrm{Lu}, \mathrm{Ce}, \mathrm{Sm}, \mathrm{Er}$, $\mathrm{La}$ и др.). Очевидно, что помимо собственно легирования редкоземельными элементами термоэлектрические свойства легированных материалов также будут зависеть от многих других характеристик материала, таких как плотность, кристаллическая и зеренная структура, элементный состав и дефектная структура. Эти характеристики должны быть тщательно оптимизированы в процессе изготовления термоэлектрического материала, легированного редкоземельными элементами, чтобы обеспечить максимальное увеличение его термоэлектрической добротности. Температура спекания является важным и контролируемым технологическим параметром, который может в достаточной степени влиять на термоэлектрические свойства материала путем изменения перечисленных выше характеристик.

Целью данной работы являлось установление закономерностей влияния температуры спекания на термоэлектрические свойства теллурида висмута, легированного гадолинием и соответствующего составу $\mathrm{Bi}_{1.9} \mathrm{Gd}_{0.1} \mathrm{Te}_{3}$.

\section{2. Методика эксперимента}

Для приготовления образцов $\mathrm{Bi}_{1.9} \mathrm{Gd}_{0.1} \mathrm{Te}_{3}$ был применен метод сольвотермального синтеза. Для корректного проведения эксперимента было получено 25 г исходного порошка в одном синтезе, который затем был поделен на 8 равных частей, использованных далее для одновременного холодного изостатического прессования в идентичных пресс-формах.

Для получения исходного порошка были использованы аналитически чистые химические реактивы $\mathrm{Bi}\left(\mathrm{NO}_{3}\right)_{3} \cdot 5 \mathrm{H}_{2} \mathrm{O}, \mathrm{TeO}_{2}, \mathrm{Gd}\left(\mathrm{CH}_{3} \mathrm{COO}\right)_{3} \cdot 4 \mathrm{H}_{2} \mathrm{O}, \mathrm{NaOH}$, поли(1-этенилпирролидин-2-он), этан-1,2-диол (этиленгликоль). Вначале нитрат висмута, оксид теллура и ацетат гадолиния, взятые в стехиометрическом соотношении, растворяли в смеси этиленгликоля $\left(450 \mathrm{~cm}^{3}\right)$ и гидроксида натрия (15г) при постоянном перемешивании с помощью магнитной мешалки. После полного 
Таблица 1. Параметры кристаллической решетки $а, c$ и элементный состав образцов соединения $\mathrm{Bi}_{2-x} \mathrm{Gd}_{x} \mathrm{Te}_{3-y}$ в зависимости от температуры спекания

\begin{tabular}{|c|c|c|c|c|c|c|}
\hline \multirow{2}{*}{$\begin{array}{r}\text { Температура } \\
\text { спекания, K }\end{array}$} & \multicolumn{2}{|c|}{$\begin{array}{c}\text { Параметры } \\
\text { кристаллической решетки }\end{array}$} & \multirow{2}{*}{$\begin{array}{c}\text { Объем элементарной } \\
\text { ячейки, } \AA^{3}\end{array}$} & \multicolumn{3}{|c|}{ Элементный состав } \\
\hline & $a, \AA$ & $c, \AA$ & & $\mathrm{Bi}$, ат\% $\%$ & $\mathrm{Gd}, \mathrm{aT} \%$ & Те, ат\% \\
\hline 750 & 4.351 & 30.276 & 496.413 & 38.7 & 2.2 & 59.1 \\
\hline 780 & 4.365 & 30.353 & 500.763 & 38.8 & 2.1 & 59.1 \\
\hline 810 & 4.369 & 30.422 & 502.806 & 38.8 & 2.2 & 59.0 \\
\hline 840 & 4.385 & 30.454 & 507.071 & 40.4 & 2.3 & 56.0 \\
\hline
\end{tabular}

растворения исходных веществ в реакционную смесь добавляли поли(1-этенилпирролидин-2-он) (молекулярная масса $\left.M_{r}=12000\right)$ и оставляли перемешиваться еще на 30 мин. Затем автоклав герметизировали и нагревали до $190^{\circ}$ С. Синтез проводили в течение 18 ч.

После остывания автоклава до комнатной температуры полученный темно-серый порошок отделяли методом центрифугирования и промывали деионизированной водой до значения $p H=7$, а затем несколькими порциями горячего этилового спирта. Сушили полученный порошкообразный материал в вакуумном сушильном шкафу при температуре $80^{\circ} \mathrm{C}$ в течение 8 ч.

Компактирование порошков и получение объемных материалов осуществляли методом холодного изостатического прессования (пресс EPSI) при давлении 220 МПа и выдержке под давлением в течение $120 \mathrm{c}$. Отжиг проводили в трубчатой печи Nobertherm RS 80/300/13 в потоке аргона при различных температурах $(750,780,810$ и $840 \mathrm{~K})$ в течение 2 ч.

Рентгенофазовый анализ (РФА) осуществляли при помощи рентгеновского дифрактометра Rigaku SmartLab ( $\mathrm{Cu} K_{\alpha}$-излучение).

Количественный элементный состав объемных материалов определяли методом оптико-эмиссионной спектроскопии с индукционно связанной плазмой параллельного действия (спектрометр ICPE-9000 Shimadzu).

Удельное электрическое сопротивление $(\rho)$ и коэффициент Зеебека $(S)$ измеряли с использованием установки ZEM-3.

Тип проводимости, концентрацию и подвижность носителей заряда определяли с помощью измерения эффекта Холла (система Cryogenic Free).

Установку ТС-1200 применяли для определения теплопроводности методом лазерной вспышки.

\section{3. Экспериментальные результаты}

Методом РФА было установлено, что синтезированный порошок является однофазным и имеет кристаллическую решетку, характерную для $\mathrm{Bi}_{2} \mathrm{Te}_{3}$ (пространственная группа симметрии $R \overline{3} m$ ). Содержание гадолиния в исходном порошке, определенное методом ICPE, соответствовало номинальному составу $\mathrm{Bi}_{1.9} \mathrm{Gd}_{0.1} \mathrm{Te}_{3}$.
Исходный порошок был компактирован методом холодного изостатического прессования и подвергнут спеканию в инертной среде аргона при в течение 2 ч при температурах 750, 780, 810 и $840 \mathrm{~K}$.

На рис. 1 представлены дифрактограммы образцов, спеченных при различных температурах. Как и в случае исходного порошка, все дифрактограммы соответствуют фазе с кристаллической решеткой $R \overline{3} m$, наличия посторонних фаз выявлено не было. Анализ дифрактограмм с помощью метода Ритвельда позволил определить параметры кристаллической решетки для всех полученных образцов (рис. 1, табл. 1).

Из рис. 1 видно, что при увеличении температуры спекания положение пиков смещается в область малых углов, что свидетельствует об увеличении параметров кристаллической решетки (штриховой линией отмечено положение пика (015) для $\mathrm{Bi}_{2} \mathrm{Te}_{3}$ (PDF\#01-0894302)). Обнаруженное изменение параметров кристаллической решетки может быть связано с испарением теллура при высоких температурах [26-28], что подтверждается результатами количественного элементного анализа методом ICPE (табл. 1), и образованием антиструктурных дефектов [29,30]. При образовании антиструктурных дефектов типа $\mathrm{Bi}_{\text {Tе }}$ увеличение параметров кристаллической решетки связано с различием радиусов атомов висмута и теллура: $r_{a}(\mathrm{Bi})=0.212 \mathrm{Hм}$, $r_{a}(\mathrm{Te})=0.152 \mathrm{Hм}[31,32]$.

Рассмотрим изменение концентрации и подвижности электронов в исследуемых образцах в зависимо-

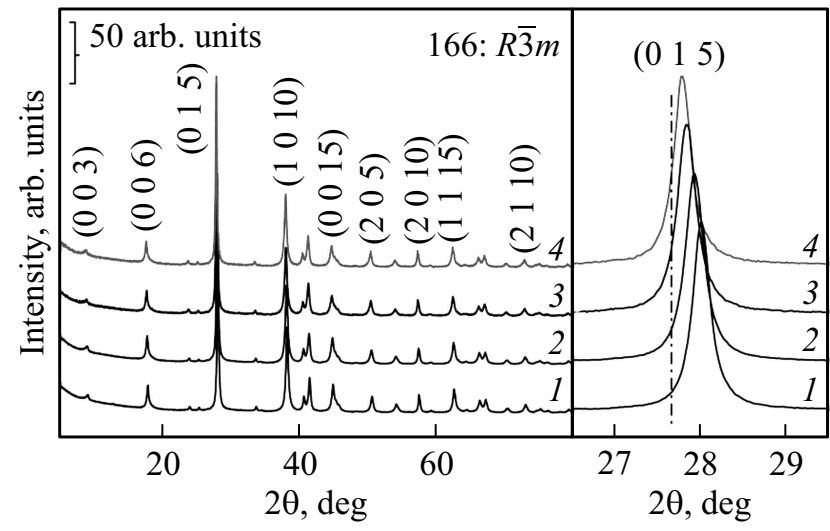

Рис. 1. Дифрактограммы объемных образцов, спеченных при температурах 750 (1), 780 (2) 810 (3) и $840 \mathrm{~K}$ (4). 
сти от температуры спекания. Как известно, тип и концентрация основных носителей заряда в теллуриде висмута в значительной мере определяются наличием точечных дефектов и их концентрацией [14-16]. В процессе высокотемпературного спекания материалов на основе $\mathrm{Bi}_{2} \mathrm{Te}_{3}$ происходит испарение теллура вследствие аномально высокого давления насыщенных паров теллура [26-28,33], так как энергия испарения Те (52.55кДж/моль) намного ниже, чем $\mathrm{Bi}$ (104.80 кДж/моль) [34]. В случае отклонения от стехиометрии в сторону дефицита теллура возможно образование трех типов точечных дефектов [14,35].

- Вакансии теллура, $V_{\mathrm{Te}}^{\bullet \bullet}$. Образование вакансий сопровождается появлением двух электронов на каждую вакансию в соответствии с выражением

$$
\mathrm{Bi}_{2} \mathrm{Te}_{3}=2 \mathrm{Bi}_{\mathrm{Bi}}^{\times}+2 \mathrm{Te}_{\mathrm{Te}}^{\times}+\mathrm{Te}_{(g)} \uparrow+V_{\mathrm{Te}}^{\bullet \bullet}+2 e^{\prime} .
$$

- Антиструктурные дефекты типа $\mathrm{Bi}_{\mathrm{Te}}^{\prime}$. В условиях дефицита теллура и из-за небольшой разности электроотрицательностей висмута и теллура, $\chi(\mathrm{Bi}=2.02$, $\chi(\mathrm{Te})=2.01[36,37]$, легко будут образовываться антиструктурные дефекты $\mathrm{Bi}_{\mathrm{Te}}^{\prime}$. Образование антиструктурных дефектов $\mathrm{Bi}_{\mathrm{Te}}^{\prime}$ приводит к закономерному образованию вакансий висмута, причем отношение $V_{\mathrm{Bi}}^{\prime \prime \prime} / V_{\mathrm{Te}}^{\bullet \bullet}$ всегда равно 2/3 [35]. Формирование каждого антиструктурного дефекта $\mathrm{Bi}_{\mathrm{Te}}^{\prime}$ сопровождается образованием двух дырок на каждый дефект в соответствии с выражением

$$
\begin{aligned}
5 \mathrm{Bi}_{2} \mathrm{Te}_{3} & =8 \mathrm{Bi}_{\mathrm{Bi}}^{\times}+10 \mathrm{Te}_{\mathrm{Te}}^{\times}+5 \mathrm{Te}_{g} \uparrow \\
& +\left(2 V_{\mathrm{Bi}}^{\prime \prime \prime}+3 V_{\mathrm{Te}}^{\bullet \bullet}\right)+2 \mathrm{Bi}_{\mathrm{Te}}^{\prime}+2 h^{\bullet} .
\end{aligned}
$$

В работе [38] сделаны оценки энергии формирования антиструктурных дефектов как функции полярности связи (разности электроотрицательности), которые приводят к заключению, что введение в кристаллическую решетку теллурида висмута легирующих примесей с электроотрицательностью, отличной от значений для $\mathrm{Bi}$ и Те, обусловливает небольшое изменение полярности связей. Однако даже этого изменения достаточно для подавления процесса образования антиструктурных дефектов. Такими легирующими элементами могут быть различные лантаноиды и, в частности, гадолиний $(\chi(\mathrm{Gd})=1.2)[35,36]$.

Учитывая все сказанное, а также результаты РФА и ICPE, можно предположить, что в интервале температур спекания от 750 до $840 \mathrm{~K}$ имеет место испарение теллура с закономерным образованием вакансий $V_{\mathrm{Te}}^{\bullet \bullet}$ и последующим образованием антиструктурных дефектов типа $\mathrm{Be}_{\mathrm{Te}}^{\prime}$.

Из результатов исследования эффекта Холла следует, что увеличение температуры спекания от 750 до $780 \mathrm{~K}$ приводит к увеличению концентрации носителей заряда от $n=4.3 \cdot 10^{19}$ до $4.9 \cdot 10^{19} \mathrm{~cm}^{3}$, при дальнейшем увеличении температуры концентрация носителей заряда остается постоянной. Изменение холловской подвижности $\mu$ электронов является более сложным. Очевидно,

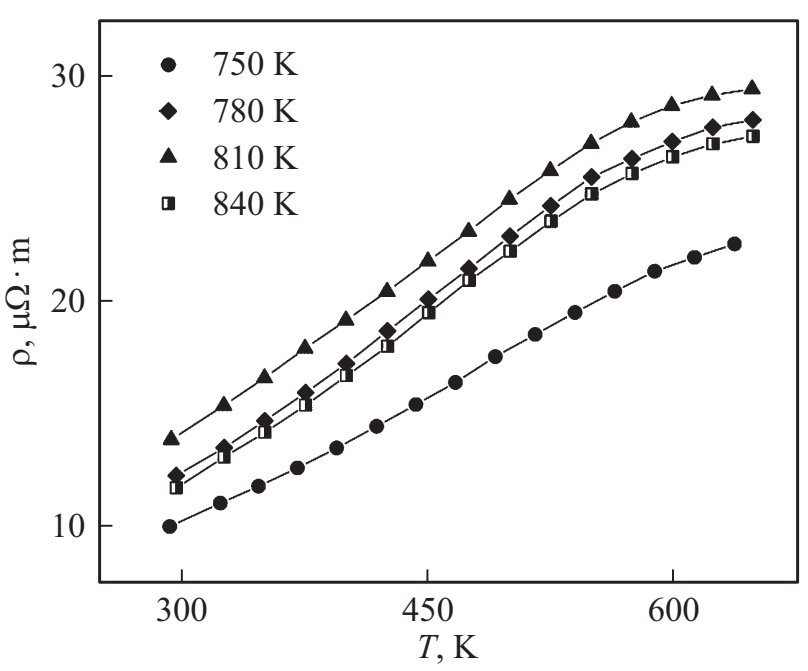

Рис. 2. Температурные зависимости удельного электрического сопротивления исследуемых образцов.

что как изменение $n$, так и изменение $\mu$, зависят от особенностей формирования и концентрации вакансий $V_{\mathrm{Te}}^{\bullet \bullet}$ и антиструктурных дефектов $\mathrm{Bi}_{\mathrm{Te}}^{\prime}$ в процессе спекания образцов. Также необходимо учитывать возможное изменение зеренной структуры при высоких температурах (межзеренные границы могут служить дополнительными центрами рассеяния для электронов). Определение конкретных механизмов влияние различных дефектов на изменение концентрации и подвижности электронов в зависимости от температуры спекания будет являться следующим этапом исследования.

Температурные зависимости удельного электрического сопротивления представлены на рис. 2. Для всех исследуемых образцов наблюдается рост удельного электрического сопротивления с ростом температуры, что характерно для вырожденных полупроводников, в частности для теллурида висмута, легированного различными лантаноидами [17-25]. Минимальное удельное электрическое сопротивления для образца $\mathrm{Bi}_{2-x} \mathrm{Gd}_{x} \mathrm{Te}_{3-y}$, спеченного при $750 \mathrm{~K}$, обеспечивается за счет достижения максимального значения подвижности электронов, хотя концентрация электронов для этого образца минимальна (табл. 2). При дальнейшем увеличении температуры спекания изменение концентрации носителей

Таблица 2. Концентрации $n$ и подвижность $\mu$ основных носителей заряда в образцах соединения $\mathrm{Bi}_{2-x} \mathrm{Gd}_{x} \mathrm{Te}_{3-y}$, спеченных при разных температурах

\begin{tabular}{c|c|c}
\hline $\begin{array}{c}\text { Температура } \\
\text { спекания, } \mathrm{K}\end{array}$ & $n, 10^{19} \mathrm{~cm}^{-3}$ & $\mu, \mathrm{cm}^{2} / \mathrm{B} \cdot \mathrm{c}$ \\
\hline 750 & 4.3 & 144 \\
780 & 4.9 & 104 \\
810 & 5.0 & 91 \\
840 & 4.9 & 110
\end{tabular}

Физика и техника полупроводников, 2019, том 53, вып. 5 


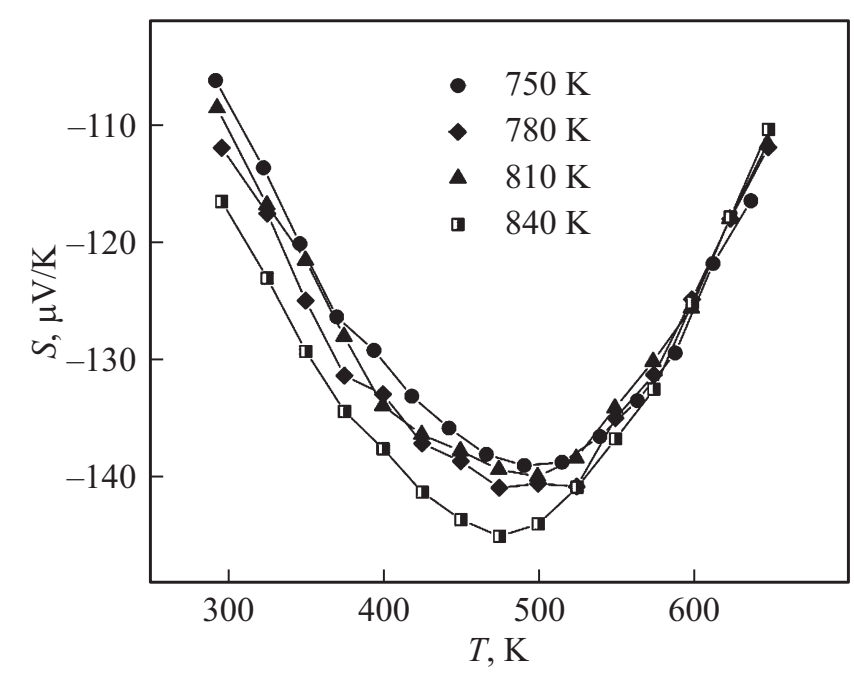

Рис. 3. Температурные зависимости коэффициента Зеебека исследуемых образцов.

заряда является незначительным и изменение удельного электрического сопротивления коррелирует с изменением подвижности электронов.

Также необходимо отметить, что для всех исследуемых образцов выше температуры $\sim 490 \mathrm{~K}$ наблюдается перегиб на температурной зависимости $\rho$, соответствующий замедлению роста $\rho$ при повышении температуры. Появление этого изгиба может быть связано с наступлением собственной проводимости, сопровождающейся термической генерацией электронно-дырочных пар, что повышает концентрацию носителей заряда и, следовательно, уменьшает удельное электрическое сопротивление.

Температурные зависимости коэффициента Зеебека для исследованных образцов представлены на рис. 3 . Поскольку основными носителями заряда во всех образцах являются электроны, коэффициент Зеебека имеет отрицательный знак. Также на всех кривых $S(T)$ наблюдается минимум, характерный для теллурида висмута, легированного различными редкоземельными элементами [17,34,37-40]. Появление этого минимума связано с биполярным вкладом при высоких температурах, обусловленным собственной проводимостью. В этом случае, в дополнение к электронам, являющимся основными носителями заряда, будут появляться дырки, причем для дырочной проводимости знак коэффициента Зеебека будет уже положительный. Следует заметить, что температура минимума на зависимости $S(T)$ соответствует температуре перегиба на зависимости $\rho(T)$, что подтверждает предположение о наступлении собственной проводимости в исследуемых образцах при температуре $\gtrsim 490$ К. Для всех исследуемых образцов коэффициент Зеебека имеет близкие значения, что свидетельствует о слабом влиянии на него температуры спекания.

Температурные зависимости теплопроводности образцов, спеченных при разных температурах, показаны на рис. 4. Температурные зависимости удельной теплопроводности $\kappa$ образцов, спеченных при температурах 750, 780 и $810 \mathrm{~K}$ практически совпадают. Для образца, спеченного при температуре $840 \mathrm{~K}$, удельная теплопроводность увеличивается. Необходимо проведение дополнительных исследований для выяснения причин такого поведения.

Следует также отметить появление минимума на кривых $\kappa(T)$ при температуре $\sim 400 \mathrm{~K}$. Появление этого минимума обусловлено изменением механизма теплопроводности. Для исследуемого интервала температур уменьшение $\kappa$ с ростом температуры в области ниже температуры минимума может быть связано с уменьшением длины свободного пробега фононов, а рост $\kappa$ в области выше температуры минимума - с появлением биполярного вклада в полную теплопроводность. В процессе биполярной теплопроводности на горячем торце

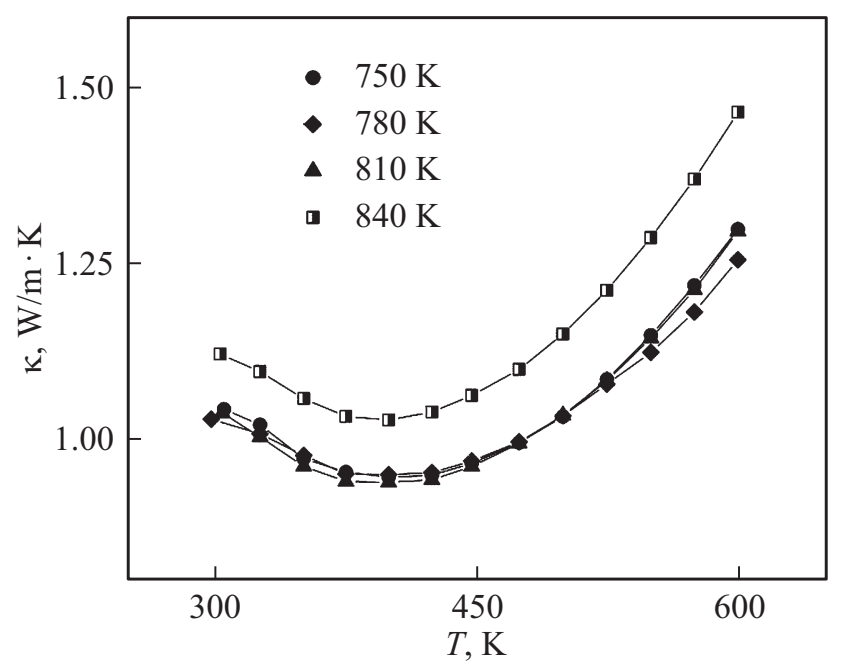

Рис. 4. Температурные зависимости удельной теплопроводности исследуемых образцов.

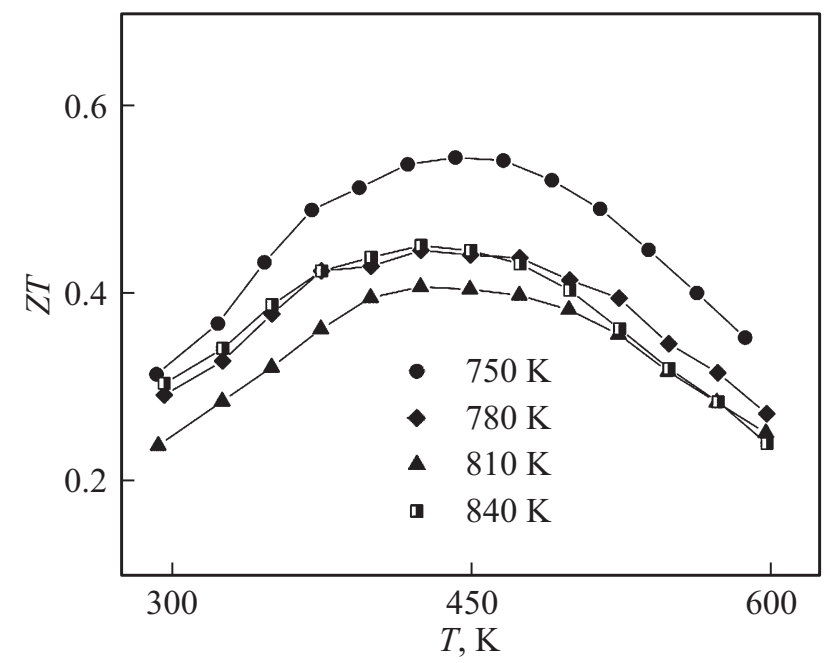

Рис. 5. Температурные зависимости термоэлектрической добротности исследуемых образцов. 
образца будут термически возбуждаться электроннодырочные пары, которые затем диффундируют к холодному торцу, где рекомбинируют, причем при рекомбинации одной электронно-дырочной пары выделяется энергия, примерно равная ширине запрещенной зоны.

Температурные зависимости термоэлектрической добротности для всех исследуемых образцов представлены на рис. 5. Наибольшее значение $Z T$ достигается для образца, спеченного при температуре $750 \mathrm{~K}(Z T \approx 0.55$ при $440 \mathrm{~K})$, потому что именно этот образец имеет минимальные значения удельного электрического сопротивления и полной теплопроводности. Уменьшение термоэлектрической добротности в области выше $\gtrsim 450 \mathrm{~K}$ связано с наступлением собственной проводимости в исследуемых образцах.

\section{4. Заключение}

Изучено влияние температуры спекания на особенности кристаллической структуры (параметры элементарной ячейки), фазовый состав и термоэлектрические свойства образцов соединения $\mathrm{Bi}_{1.9} \mathrm{Gd}_{0.1} \mathrm{Te}_{3}$. Благодаря оптимальному сочетанию удельного электросопротивления, коэффициента Зеебека и общей теплопроводности наибольшее значение термоэлектрической добротности $(Z \approx 0.55)$ наблюдалось для образца, спеченного при температуре $750 \mathrm{~K}$. Возникновение собственной проводимости, наблюдаемое при изучении термоэлектрических свойств образцов в области $\gtrsim 490 \mathrm{~K}$, негативно сказывается на возможности дальнейшего повышения ZT, поскольку термическое возбуждение электронно-дырочных пар снижает коэффициент Зеебека и увеличивает теплопроводность.

Исследование выполнено при финансовой поддержке РФФИ в рамках научного проекта № 18-32-00415.

\section{Список литературы}

[1] G.S. Nolas, J. Sharp, H.J. Goldsmid. Thermoelectrics Basic Principles and New Materials Developments (Berlin, Springer, 2001).

[2] H.J. Goldsmid. Materials, 7, 2577 (2014).

[3] H. Kitagawa, T. Nagamori, T. Tatsuta, T. Kitamura, Y. Shinohara, Y. Noda. Scripta Mater., 49, 309 (2003).

[4] D.B. Hyun, T. S. Oh, J.S. Hwang, J.D. Shim, N.V. Kolomoets. Scripta Mater., 40, 49 (1998).

[5] S. Miura, Y. Satob, K. Fukuda, K. Nishimura, K. Ikeda. Mater. Sci. Eng. A, 277, 244 (2000).

[6] O. Ivanov, O. Maradudina, R. Lyubushkin. J. Alloys Compd., 586, 679 (2014).

[7] W. Liu, X. Yan, G. Chen, Z. Ren. Nano Energy, 1, 42 (2012).

[8] Y. Li, J. Jiang, G. Xu, W. Li, L. Zhou, Y. Li, P. Cui. J. Alloys Compd., 480, 954 (2009).

[9] S.S. Kim, S. Yamamoto, T. Aizawa. J. Alloys Compd., 375, 107 (2004).

[10] Y. Morisaki, H. Araki, H. Kitagawa, M. Orihashi, K. Hasezaki, K. Kimura. Mater. Trans., 46, 2518 (2005).
[11] X.K. Duan, K.G. Hu, D.H. Ma, W.N. Zhang, Y.Z. Jiang, S.C. Guo. Rare Metals, 34, 770 (2015).

[12] P. Srivastava, K. Singh. Mater. Lett., 136, 337 (2014).

[13] B. Jarivala, D. Shah, N.M. Ravindra. J. Electron. Mater., 44, 1509 (2015).

[14] Y. Pan, T.R. Wei, C.F. Wu, J.F. Li. J. Mater. Chem. C, 3, 10583 (2015).

[15] L. Hu, T. Zhu, X. Liu, X. Zhao. Adv. Funct. Mater., 24, 5211 (2014).

[16] J. Suh, K.M. Yu, D. Fu, X. Liu, F. Yang, J. Fan, D.J. Smith, Y.H. Zhang, J.K. Furdyna, C. Dames, W. Walukiewicz, J. Wu. Adv. Mater., 27, 3681 (2015).

[17] J. Yang, F. Wu, Z. Zhu, L. Yao, H. Song, X. Hu. J. Alloys Compd. 619, 401 (2015).

[18] X.H. Ji, X.B. Zhao, Y.H. Zhang, B.H. Lu, H.L. Ni. J. Alloys Compd., 387, 282 (2005).

[19] F. Wu, H. Song, J. Jia, X. Hu. Prog. Nat. Sci. Mater. Int., 23, 408 (2013).

[20] F. Wu, W. Shi, X. Hu. Electron. Mater. Lett., 11, 127 (2015).

[21] X.H. Ji, X.B. Zhao, Y.H. Zhang, B.H. Lu, H.L. Ni. Mater. Lett., 59, 682 (2005).

[22] F. Wu, H.Z. Song, J.F. Jia, F. Gao, Y.J. Zhang, X. Hu. Phys. Status Solidi A, 210, 1183 (2013).

[23] W.Y. Shi, F. Wu, K.L. Wang, J.J. Yang, H.Z. Song, X.J. Hu. Electron. Mater., 43, 3162 (2014).

[24] X.B. Zhao, Y.H. Zhang, X.H. Ji. Inorg. Chem. Commun., 7, 386 (2004).

[25] O. Ivanov, M. Yaprintsev, R. Lyubushkin, O. Soklakova. Scr. Mater., 146, 91 (2018).

[26] S.A. Humphry-Baker, C.A. Schuh. Nano Energy, 36, 223 (2017).

[27] J. Lee, J. Kim, W. Moon, A. Berger, J. Lee. J. Phys. Chem. C, 116, 19512 (2012).

[28] J. Lee, A. Berger, L. Cagnon, U. Gosele, K. Nielsch, J. Lee. Phys. Chem. Chem. Phys., 12, 15247 (2010).

[29] P. Losták, C. Drasar, D. Bachan, L. Benes, A. Krejcová. Rad. Eff. Def. Sol., 165, 211 (2010).

[30] Ю.Е. Калинин, М.А. Каширин, В.А. Макагонов, С.Ю. Панков, А.В. Ситников. ФТТ, 59 (1), 23 (2017).

[31] D.C. Ghosh, R. Biswas. Int. J. Mol. Sci., 3, 87 (2002).

[32] M.V. Putz, N. Russo, E. Sicilia. J. Phys. Chem. A, 107, 5461 (2003).

[33] N.T. Nghi, A.L. Usiikans, T.A. Cherepanova. Cryst. Res. Technol., 21, 367 (1986)

[34] M. Yaprintsev, R. Lyubushkin, O. Soklakova, O. Ivanov. J. Electron. Mater., 47, 1362 (2018).

[35] Z. Stary, J. Horak, M. Stordeur, M. Stolzer. J. Phys. Chem. Solids, 49, 29 (1988).

[36] L. Pauling. J. Am. Chem. Soc., 54, 3570 (1932).

[37] J.C.A. Boeyens. Z. Naturforsch., 63b, 199 (2008).

[38] J. Horak, K. Cermak, L. Koudelka. J. Phys. Chem. Solids, 47, 805 (1986).

[39] M. Yaprintsev, R. Lyubushkin, O. Soklakova, O. Ivanov. Rare Metals, 37, 642 (2018).

[40] L. Yao, F. Wu, X.X. Wang, R.J. Cao, X.J. Li, X. Hu, H.Z. Song. J. Eelectron. Mater., 45, 3053 (2016).

Редактор Л.В. Шаронова

Физика и техника полупроводников, 2019, том 53, вып. 5 


\section{Sintering temperature effect on thermoelectric properties of the $\mathrm{Bi}_{1.9} \mathbf{G d}_{0.1} \mathrm{Te}_{3}$ compound}

M.N. Yapryntsev ${ }^{\mathbf{1}}$, A.E. Vasiliev ${ }^{\mathbf{1}}$, O.N. Ivanov $^{\mathbf{1 , 2}}$

${ }^{1}$ Belgorod National Research University, 308015 Belgorod, Russia

${ }^{2}$ Belgorod State Technological University named after V.G. Shukhov, 308012 Belgorod, Russia

The peculiarities of sintering temperature $(750,780$, 810 and $840 \mathrm{~K}$ ) effect on the elemental composition, the crystal lattice parameters, the specific electrical resistivity, the Seebeck coefficient, the total thermal conductivity and the thermoelectric figure-of-merit for the $\mathrm{Bi}_{1.9} \mathrm{Gd}_{0.1} \mathrm{Te}_{3}$ compound have been studied. It was found that intensive evaporation of $\mathrm{Te}$ occurs during the high-temperature sintering resulting in various defects forming (like vacancies and antisite defects). These defects can effect on both content and mobility of the majority carriers (electrons). The specific electrical resistivity was found to be sensitive to the sintering temperature, whereas the sintering temperature effect on the Seebeck coefficient and the total thermal conductivity was weak enough. The highest thermoelectric figure-of-merit $(Z T \approx 0.55)$ was observed for the sample sintered at $750 \mathrm{~K}$. 